Bucknell University

Bucknell Digital Commons

\title{
Rats' Learned Preferences for Flavors Encountered Early or Late in a Meal Paired with the Postingestive Effects of Glucose
}

Kevin P. Myers

Bucknell University, kmyers@bucknell.edu

Margaret C. Whitney

Follow this and additional works at: https://digitalcommons.bucknell.edu/fac_journ

\section{Recommended Citation}

Myers, Kevin P. and Whitney, Margaret C.. "Rats' Learned Preferences for Flavors Encountered Early or Late in a Meal Paired with the Postingestive Effects of Glucose." Physiology \& Behavior (2011) : 466-474.

This Article is brought to you for free and open access by the Faculty Scholarship at Bucknell Digital Commons. It has been accepted for inclusion in Faculty Journal Articles by an authorized administrator of Bucknell Digital Commons. For more information, please contact dcadmin@bucknell.edu. 


\title{
Rats' learned preferences for flavors encountered early or late in a meal paired with the postingestive effects of glucose
}

\author{
Kevin P. Myers ${ }^{\mathrm{a}, \mathrm{b}, \mathrm{c}, *}$, Margaret C. Whitney ${ }^{\mathrm{b}}$ \\ a Department of Psychology, Bucknell University, USA \\ ${ }^{\mathrm{b}}$ Program in Animal Behavior, Bucknell University, USA \\ c Program in Neuroscience, Bucknell University, USA
}

\section{A R T I C L E I N F O}

\section{Article history:}

Received 13 August 2010

Received in revised form 9 December 2010

Accepted 13 December 2010

\section{Keywords:}

Flavor-nutrient conditioning

Food preferences

Learning

Reward

Calories

Glucose

Hunger

Dessert

\begin{abstract}
A B S T R A C T
Rats learn to prefer flavors that are followed by postingestive effects of nutrients. This experiment investigated whether the timing of a flavor (specifically, in the first or second half of the meal) influences learning about that flavor. Stronger learning about earlier or later flavors would indicate when the rewarding postingestive effects of nutrients are sensed. Rats with intragastric (IG) catheters drank saccharin-sweetened, calorically-dilute solutions with distinct flavors added, accompanied by IG infusion of glucose (+ sessions) or water ( - sessions). In both types of sessions, an "Early" flavor was provided for the first $8 \mathrm{~min}$ and a "Late" flavor for the last $8 \mathrm{~min}$. Thus, rats were trained with Early $(+)$ and Late $(+)$ in high-calorie meals, and Early $(-)$ and Late $(-)$ in low-calorie meals. Strength of the learned preference for $\operatorname{Early}(+)$ and Late $(+)$ was then assessed in a series of two-bottle choice tests between $\operatorname{Early}(+)$ vs. $\operatorname{Early}(-)$, Late $(+)$ vs. $\operatorname{Late}(-)$, Early $(+)$ vs. Late $(+)$, and $\operatorname{Early}(-)$ vs. Late $(-)$. Rats preferred both Early $(+)$ and Late $(+)$ over the respective $(-)$ flavors. But Early $(+)$ was only preferred when rats were tested hungry. Late $(+)$ was preferred when rats were tested hungry or recently satiated. This indicates qualitatively different associations learned about flavors at different points in the meal. While not supporting the idea that postingestive effects become most strongly associated with later-occurring ("dessert") flavors, it does suggest a reason dessert flavors may remain attractive in the absence of hunger.
\end{abstract}

(c) 2010 Elsevier Inc. All rights reserved.

\section{Introduction}

Food choice and meal size are fundamentally based on sensory evaluation of foods. What an individual selects to eat and how much one eats depend strongly on one's likes and dislikes for the tastes and flavors of the various available (or obtainable) foods. But mammals possess only a few 'innate' taste preferences and aversions, and for the most part reactions to food flavors are established by learning, especially learned associations between the flavor of a food and the consequences of eating it [1-4].

One powerful way that learning alters flavor preferences is flavornutrient conditioning, whereby a flavor becomes more positively evaluated because it has been paired with positive caloric consequences. This is viewed as Pavlovian conditioning, with the flavor (CS) predicting the postingestive actions of nutrients (US). The learned flavor-nutrient association can lead to strong preference (choice of that flavor over others) and acceptance (increased absolute intake) $[5,6]$. The ability to learn that some flavors signal positive postingestive consequences presumably evolved as an adaptation for efficient foraging in generalist animals. But in the modern human

\footnotetext{
* Corresponding author. Department of Psychology, Bucknell University, Lewisburg

PA 17837, USA. Tel.: +1 570577 3493; fax: +1 5705777007.

E-mail address: kmyers@bucknell.edu (K.P. Myers).
}

environment, Pavlovian processes like flavor-nutrient conditioning may contribute to overeating and weight gain by biasing selection towards calorically dense foods and stimulating intake of overly large meals. Thus understanding the properties and functions of this learning mechanism illuminates the basic organization of appetite, and also has potential relevance for effecting healthy eating behavior.

A useful model for studying flavor-nutrient conditioning in the laboratory involves rats consuming a distinctly flavored solution (CS) by mouth while the US (e.g., glucose solution or other nutrient) is infused into the stomach via an intragastric (IG) catheter [7]. This method for pairing a flavor and nutrient ensures that the rats are learning to associate the flavor CS with the postingestive consequences of the US acting post-orally, instead of associating the CS flavor with the reward value of the nutrient's inherently attractive taste. (Such 'flavor-flavor' or 'flavor-taste' associations can also establish learned preferences for CS flavors, but are mechanistically and psychologically distinct from flavor-nutrient learning $[1,8,9]$.) The IG infusion method permits studying flavor-nutrient conditioning in isolation from other types of learning that alter food choice.

The experiment reported here was designed to investigate formation of flavor-nutrient associations at different points of time within a meal. Specifically, in meals with multiple flavors consumed in succession, would the earlier or later flavor become differentially preferred based on selective association with postingestive consequences? We believe that 
this is an important variable to investigate because the relative strength of learned preferences for flavors encountered at different points in the meal can help elucidate the timing of the physiological events that produce the 'rewarding' signal triggered by the postingestive effects of nutrients.

In a typical flavor-nutrient conditioning experiment, rats are trained with a single flavor per session, either during brief sessions (e.g., $30 \mathrm{~min}$ ) that can be thought of as single meals, or longer sessions (e.g., 4-24 h) during which subjects self-initiate multiple meals [7]. In either case, training alternates between sessions with a flavor (CS+) paired with a caloric US, and sessions with a different flavor (CS-) unpaired with calories (usually a control IG infusion of plain water). This strategy equates familiarity with the two CS flavors (at least in terms of number of sessions) so that subsequent preference is not biased by a 'mere exposure' effect. After training, the critical test of learning is a two-bottle choice between the CS+ vs. CS - flavors in the absence of the nutrient US. Rats trained in such a fashion routinely show overwhelming preference for the $\mathrm{CS}+$. Because the preference is evident even when the nutrient US is not delivered, it shows that evaluation of the flavor itself has been altered by learning. But a key feature relevant to our present study is that flavor-nutrient conditioning experiments routinely train rats with meals of only a single flavor at a time. Little is known about how flavor-nutrient conditioning operates when individuals experience multiple flavors within a single meal.

A notable exception to this alternating-sessions design is a study in which rats had simultaneous access to a CS+ flavor paired with IG carbohydrate infusion and a CS - flavor paired with IG water [10]. Almost all rats did learn to preferentially consume the CS+ flavor within the first few days of training, and did not 'erroneously' learn to associate the CS - with the nutrient that accompanied the CS+. Yet this result does not reflect an ability to rapidly discriminate which of the two flavors within an individual meal was correlated with the nutrient. The authors of that study point out that although rats had the opportunity to consume both flavors within a drinking bout, they typically did not, instead tending to distribute their drinking into temporally segregated bouts of one flavor at a time. It remains largely unknown how or what rats may learn when the postingestive actions of macronutrients are preceded by multiple distinct flavors.

To begin investigating that general issue, we designed the present experiment to determine what pattern of preferences rats would learn when they experienced meals consisting of two distinct flavors in succession, in this case both accompanied by nutrition. As already mentioned, our first main rationale for this research is that despite considerable evidence for flavor-nutrient conditioning, the exact physiological event that acts as the US for the learning is unknown. It has sometimes been called "flavor-calorie" learning, but caloric content per se does not describe the US, since different macronutrients are differentially effective even when equated for caloric density (summarized in [11]). For example, glucose and fructose are each common dietary monosaccharides, but rats do not readily learn to prefer fructose-paired flavors the way they do with glucose-paired flavors [12]. Dietary fat can serve as an effective US, but preference for a flavor paired with fat requires more training and is ultimately weaker than preference for a glucose-paired flavor [13]. The relative effectiveness of different macronutrients as a US might reflect their different metabolic fates, or perhaps different speeds at which different macronutrients are handled. We believe that our present experiment can be informative as another approach to identifying the postingestive US signal, by indicating when it begins to manifest itself during or after the meal.

We are not the first to consider the temporal relationship between the flavor CS and postingestive US in flavor-nutrient learning. But most work on that question has come from a conceptually different direction, interposing a delay between flavor and nutrient to study how long after a flavor CS the US can be delayed yet still support learning. This follows the assumption that in a normal meal the postingestive US that supports flavor-nutrient learning is not sensed until quite some time after nutrients have begun to arrive in the GI tract, perhaps not even until after the meal has ended (which is in fact the rationale for the 'reverse order' method for experimentally training flavor-nutrient associations [14]).

Indeed rats are capable of flavor-nutrient learning with a long CSUS delay interposed [15]. But in the present experiment we take the opposite approach, tracking how soon after a meal begins the postingestive US may be acting. This is inferred by determining whether or not rats learn to prefer a flavor that is only experienced at the beginning of a nutritive meal, and if so, whether that preference is weaker or stronger than for a flavor occurring at the end of a meal. A well-established principle of conditioning is that temporal contiguity between CS and US influences learning, such that (other things being equal) a CS occurring shortly before the US should be learned more readily than a CS occurring longer before the US.

This temporal contiguity principle is known to influence learned flavor aversion based on postingestive illness [16,17], and some evidence suggests its relevance to flavor-nutrient conditioning as well. For example, when rats consume a flavor CS before consuming a nutritive carbohydrate US, the taste of the carbohydrate itself occurs closer in time to its postingestive effects, and appears to be treated as a CS that interferes between the nominal 'CS' flavor and the postingestive US [15] (and see also [18]). Therefore, if rats routinely experience different flavors early and late in a nutritive meal, the resulting learned preference(s) should indicate when the US is acting. A stronger preference for the later flavor should be learned if the postingestive US has its onset only after the meal has ended. A strong preference, indeed perhaps even a stronger preference, for the early flavor would suggest that the postingestive US has rapid onset earlier in the meal.

Another rationale for initiating this line of research is to investigate the possibility of timing effects in flavor-nutrient conditioning that establish special status for flavors that routinely appear in desserts. Since flavor-nutrient conditioning may help explain the human tendency to preferentially select and over-consume calorically dense foods, it becomes relevant that humans often consume multiple foods in a meal, and perhaps more importantly that the timing of foods within a meal is often not arbitrary. Cultural cuisine rules classify some foods as entrées or desserts, and that routine timing arrangement may promote differential learning about different flavors. This has been proposed as an explanation for why flavors encountered in desserts become so desirable $[1,18]$, and could apply to why some flavors, for instance chocolate, commonly become objects of craving [19].

Some circumstantial evidence supports the idea that the postingestive nutritional US experienced following the meal might become most strongly associated with the last thing tasted. In one study [18] rats consumed separate meals of two distinct foods, one of which was always followed by a sucrose 'dessert.' The authors initially expected that the food followed by sucrose would become more preferred because of the rewarding effect of the added dessert. But in fact the opposite occurred: Preference decreased for the food eaten before the dessert. An interpretation directly relevant to the present research is that postingestive effects of nutrition may be experienced after a fairly long delay and therefore would become most strongly associated with the tastes/flavors closest to the end of the meal. In the case of a food eaten before a dessert, the nutritive consequences actually provided by the 'entrée' would instead become associated with the taste of the 'dessert.' But the authors of that study provide other viable explanations that do not involve flavor-nutrient conditioning, the most straightforward being an anticipatory contrast effect. Rats may suppress intake of the entrée food that always preceded dessert in anticipation of the coming dessert (which was already inherently better-tasting even before any learning occurred). 
Therefore, that experiment cannot necessarily be taken as evidence that those flavors occurring late in a meal become more strongly associated with the nutritive consequences of the entire meal. We believe that our current experiment would be the first direct test of that proposal.

In this experiment, rats were trained in a series of brief sessions in which flavor consumption was accompanied by IG infusion. Sessions occurred daily following overnight food deprivation. In nutritive $(+)$ sessions flavors were paired with IG glucose infusion and in nonnutritive $(-)$ sessions different flavors were paired with IG water infusion. In each type of session, an "Early" flavor was offered first and then replaced with the "Late" flavor halfway through the meal. Thus each rat was trained with four distinct flavors. Nutritive meals consisted of the Early $(+)$ flavor and then the Late $(+)$ flavor, and for comparison purposes, an equivalent number of non-nutritive meals consisted of the Early $(-)$ flavor followed by the Late $(-)$ flavor. Given that the $\operatorname{Early}(+)$ and Late $(+)$ flavors were both routinely paired with nutrition, our main question was whether rats would learn a stronger preference for one of those flavors or the other, or if preference for both would increase. Learned preferences were assessed in a series of two-bottle choice tests between each $(+)$ flavor vs. its corresponding $(-)$ flavor (i.e., $\operatorname{Early}(+)$ vs. Early $(-)$, and Late $(+)$ vs. Late $(-))$ as well as Early $(+)$ vs. Late $(+)$, and Early $(-)$ vs. Late $(-)$.

An important added feature of the two-bottle preference tests is that all tests were repeated under two different physiological conditions, "hungry" (overnight food deprived, like the training conditions) and "recently fed" (tested 90 min after chow rations). This was to investigate the possibility that learned preferences for the $\operatorname{Early}(+)$ and the Late $(+)$ flavors could be differentially statedependent. Training sessions were always conducted after overnight food deprivation, meaning that rats would be hungry when the session began. Yet presumably significant satiation would occur during $(+)$ sessions, meaning rats were actually trained with the Late $(+)$ flavor in a different state than the Early $(+)$ flavor. Furthermore the Late $(+)$ and Late $(-)$ flavors were encountered in opposite states because negligible postingestive satiation should occur during $(-)$ sessions, and it is not entirely clear which state at testing is the appropriate one for comparison. Thus all two-bottle choice tests were duplicated under both hungry and fed conditions.

\section{Methods}

\subsection{Subjects}

All animal procedures were approved by the university IACUC and were consistent with the NIH Guide for the Care and Use of Laboratory Animals. Twenty experimentally naive, adult female Sprague-Dawley $\mathrm{CD}$ rats were used. Subjects were bred in our laboratory from stock originally obtained from Ace Animals (Allentown, PA). Rats were approximately 180 days old and weighed $274 \pm 17.4 \mathrm{~g}$ (mean \pm SD) at the outset. They were housed individually in $8 \times 16 \times 10.5^{\prime \prime}$ plastic tub cages with corncob bedding. Each rat had an intragastric (IG) Silastic catheter (1.02 mm ID, $2.16 \mathrm{~mm}$ OD) surgically installed under ketamine/xylazine anesthesia (65 and $10 \mathrm{mg} / \mathrm{kg}$ ) as described in [20]. The catheter was routed from the peritoneum subcutaneously to exit between the shoulders, where it was attached to a Luer-Loc connecter that remained capped when not in use. A postoperative recovery period of at least seven days was allowed before proceeding. Beginning at that time rats were restricted to a ration of $14-15 \mathrm{~g}$ of chow daily. Tap water was available ad libitum in the rats' home cages.

\subsection{Apparatus}

Conditioning was conducted in 10 identical cylindrical test chambers, $35 \mathrm{~cm}$ high $\times 25 \mathrm{~cm}$ diameter, made of opaque plastic with a wire grid floor. When a rat was placed in its test chamber, the Luer-Loc connector on its IG catheter was connected to infusion tubing extending from a fluid swivel held above the chamber on a counterbalanced lever arm. This was connected to tubing from a $30 \mathrm{ml}$ syringe containing the fluid to be intragastrically infused, which was mounted on a syringe pump. This arrangement allows the rat unrestricted movement inside the apparatus and prevents damage to the infusion tubing and stress on the IG catheter.

The front of each chamber had two small apertures approximately $3 \mathrm{~cm}$ apart, through which the rat could access the sipper tubes of bottles carried on a motorized bottle retractor mounted on the exterior of the chamber. The bottle retractors (modified Med Associates ENV-252) could hold two $50 \mathrm{ml}$ drinking tubes side by side, but throughout training the rats were actually only given one bottle at a time. Therefore the left-right position used on the bottle retractor was systematically varied across sessions to discourage rats from developing side preferences.

Drinking from the sipper tubes was monitored by electronic contact lickometers interfaced to a computer. This computer, which also controlled the bottle retractors, counted each rat's licks and in turn individually controlled the intragastric infusion pumps. A rat's infusion pump was activated when the rat was licking, delivering the IG infusate at a rate of $0.02 \mathrm{ml} / \mathrm{s}$. This ensured that the rate and total volume of a rat's IG infusion approximately matched its oral consumption.

\subsection{Procedure}

\subsubsection{Training}

A pilot study had been conducted with separate rats to devise the session parameters. All flavors given to the rats for oral consumption were mixed in a vehicle of $2 \%$ fructose $+0.2 \%$ saccharin. This mixture was chosen because it is intrinsically highly palatable but minimally satiating, and this would encourage the rats to drink at fairly consistent rates for the duration of the brief sessions. The rationale for this was to approximately equate mere exposure to the Early and Late flavors in a session. A pilot study on drinking rates with this solution determined that when given the solution for a $16 \mathrm{~min}$ session, rats consumed approximately equivalent amounts during the first and second halves. Although the vehicle solution contained $2 \%$ fructose and therefore provided some calories, several studies have shown that fructose does not generate postingestive rewarding stimulation that produces flavor-nutrient conditioning (see $[11,12,21,22])$. Thus we believe that the fructose in the vehicle solution is a negligible consideration.

After rats had been on the daily food rationing for at least six days, but before conditioning began, rats were familiarized with the conditioning chambers in a series of daily acclimation sessions. During the first two sessions they were connected to the infusion tubing but not infused as they drank, and in the next four they were infused with water IG as they drank. Training sessions occurred once daily in the late morning.

Rats were trained using two types of sessions. In $(+)$ sessions both the Early and Late flavors were paired with IG infusion of $12 \%(\mathrm{w} / \mathrm{w})$ glucose. In ( - ) sessions the Early and Late flavors were paired with IG water. Thus each rat was trained with four CS flavors: Early $(+)$ and Late $(+)$ were paired with IG glucose, whereas Early $(-)$ and Late $(-)$ were not. The flavors used as CSs were cherry, grape, lemon-lime, and orange Kool-Aid (Kraft Foods Inc., Glenview, IL; powdered unsweetened Kool-Aid mix was dissolved in the sweet vehicle solution at a concentration of $0.05 \%$ of solution by weight). Assignment of each rat's flavor contingencies was completely counterbalanced. Rats were given only one session per day, and sessions type $(+$ or - ) alternated across days in a double-alternation sequence. The entire training phase consisted of eight $(+)$ and eight $(-)$ sessions. 
A training session began with the presentation of the Early flavor for $8 \mathrm{~min}$. Then the bottles automatically retracted and during a 4-min pause the Early bottle was switched with the Late bottle. When the 4-min pause elapsed the Late flavor was inserted and was available for $8 \mathrm{~min}$. Throughout the session, consumption of both the Early flavor and the Late flavor was accompanied by IG infusion as described above. Intake from each bottle was measured by weighing bottles immediately before and after sessions. Chow rations were given 90 min after the end of the training session daily.

\subsubsection{Two-bottle tests}

Beginning two days after the completion of the conditioning phase, rats' learned preferences for the flavors were assessed in a series of two-bottle choice tests. We had initially intended to conduct these two-bottle tests in the apparatus used for training. However, initial 'practice' tests with unflavored fructose + saccharin solutions that we conducted to familiarize rats with the two-bottle choice procedure showed that many rats had strong side preferences and would drink only from one bottle even if a sweeter solution was in the other bottle. We cannot explain this unusual behavior, since during training bottle position was alternated systematically so as not to confound with $(+)$ or $(-)$ session type. Nonetheless, because of this bias we elected to conduct the series of daily two-bottle tests in rats' home cages. This means that rats were not connected to the IG infusion system during the tests, but this is of little consequence since the rationale of the tests was to assess learned changes in evaluation of the flavors per se.

During the series of two-bottle tests, rats were tested twice daily. One 30-min test was conducted at mid-morning after overnight deprivation (the approximate time and hunger state of the training sessions). Two hours after the morning test rats received their daily chow rations. They were then given a second 30-min two-bottle test while in the "recently fed" state, beginning 90 min after chow rations were distributed. Tests were arranged so that an afternoon (fed) test never included either of the flavors that were given in that morning's (hungry) test.

To begin, rats were first acclimated to this new schedule and home cage two-bottle test procedure with two days of twice-daily practice tests. One bottle contained unflavored $1 \%$ fructose $+0.1 \%$ saccharin, while the other contained unflavored $3 \%$ fructose $+0.3 \%$ saccharin. Across testing the left-right positions was reversed. This encouraged rats to sample each bottle and allowed us to verify rats were choosing based on bottle contents and not position. During all two-bottle tests the bottles were placed simultaneously onto the wire lids of the cages so that the sipper tubes protruded into the cage, centered approximately $4 \mathrm{~cm}$ apart. Intake was measured by weighing each bottle before and after the test.

The four critical preference tests compared Early $(+)$ vs. Late $(+)$, $\operatorname{Early}(-)$ vs. Late $(-)$, Early $(+)$ vs. $\operatorname{Early}(-)$, and Late $(+)$ vs. Late $(-)$. Each test was repeated twice under hungry conditions and twice under fed conditions, with the left-right position of the flavors reversed for each repetition. The order of the four different tests across days was counterbalanced across rats, and for each rat the order was reversed in the first and second replications of the test series. The two repetitions of each test in a particular deprivation state were averaged for analysis of the results. This series of tests necessarily involves several exposures across days to each $(+)$ flavor now unaccompanied by nutrient, but we do not regard potential extinction of learned preference as a concern. Typically extinction of flavor-nutrient learning is found to decrease absolute intake of a flavor (learned acceptance) while leaving relative preference for one flavor over another (as is being measured here) intact, even after numerous extinction exposures $[23,24]$. In any case, any extinction during testing would favor a null result, so this is an inherently conservative measure of preference strength, nor could extinction accruing during testing systematically bias the results because of the complete counterbalancing of test order.

\section{Results}

\subsection{Training sessions}

There were eight training sessions of each type (+ or -) conducted across 16 days in double-alternation order. To simplify the analysis, the eight sessions of each type were condensed to four two-session blocks by averaging consecutive sessions per type. Intakes (shown in Fig. 1) were analyzed with a $2(+$ vs. - Session Type $) \times 2$ (Early vs. Late flavor $) \times 4$ (Session Blocks) repeated measures ANOVA.

In several ways the rats' behavior was similar in $(+)$ and $(-)$ sessions. In both types of sessions intakes rose across the series of training days (main effect of Session Block: $F[3,57]=38.4, p<0.01$ ) and rats consistently consumed more Early flavor than Late flavor (main effect of Early vs. Late: $F[1,19]=73.0, p<0.01$ ). Increasing intakes across sessions is primarily accounted for by increased intake of the Early flavor, not the Late flavor (Session Block $\times$ Early vs. Late interaction: $F[3,57]=26.7, p<0.01$ )
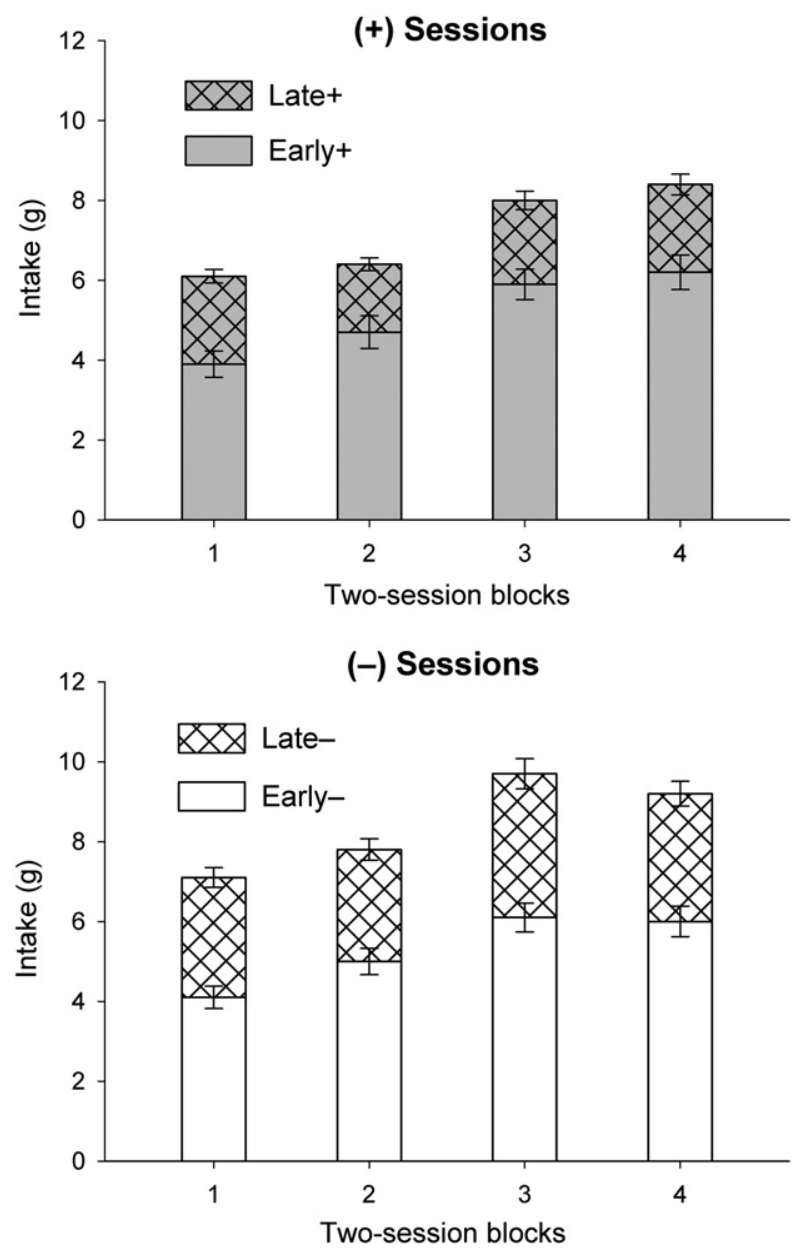

Fig. 1. Intakes in daily training sessions, showing the mean ( \pm SEM) consumption of the Early flavor (bottom portion of each bar) and the Late flavor (top portion of each bar). In $(+)$ sessions (upper panel) consumption of the $0.2 \%$ saccharin $+2 \%$ fructose vehicle with the added cue flavors was accompanied by IG $12 \%$ glucose infusion, and in ( - ) sessions (lower panel) it was accompanied by IG water infusion. In both types of sessions the Early flavor was available for $8 \mathrm{~min}$, followed by a 4 min timeout, and then the Late flavor for $8 \mathrm{~min}$. Each bar represents the average of two consecutive sessions of that type. 
But rats behaved differently in $(+)$ sessions compared to $(-)$ sessions in two ways. First, total session intakes were significantly less in $(+)$ than in $(-)$ sessions (main effect of Session Type: $F[1,19]=$ $34.6, p<0.01$ ). Second, that difference is specifically due to intakes of the Late flavors. Rats consumed similar amounts of Early $(+)$ and Early $(-)$, but significantly less Late $(+)$ than Late $(-)$ (Session Type $\times$ Early vs. Late interaction: $F[1,19]=9.51, p<0.01)$.

\subsection{Two-bottle tests}

Because four independent t-tests comprise the analysis for each deprivation state, familywise Type-I error rate was maintained at 0.05 by adjusting the threshold for statistical significance to $p<0.0125$, as per the Bonferroni correction.

\subsubsection{Two-bottle tests: Hungry}

Two-bottle choice tests revealed that, when hungry, rats preferred both of the flavors they had experienced in $(+)$ meals over the corresponding $(-)$ flavors. As shown in Fig. 2, rats preferred Early $(+)$ $\operatorname{over} \operatorname{Early}(-)$ (Fig. 2a, $t[19]=2.91, p=0.009)$ and preferred Late $(+)$ over Late $(-)$ (Fig. $2 b, t[19]=3.69, p=0.002$ ). There was no indication that preference for $\operatorname{Early}(+)$ was any weaker or stronger than the preference for Late $(+)$. The degree of preference for each $(+)$ flavor over its corresponding $(-)$ flavor was similar when expressed as a percentage of total consumption in the test (Fig. 2a and b, 66.4\% preference for $\operatorname{Early}(+)$ over $\operatorname{Early}(-)$ and $68.1 \%$ preference for Late $(+)$ over Late $(-))$ and also in terms of absolute intakes (mean $\pm \mathrm{SEM}=11.3 \pm 1.54 \mathrm{~g}$ for Early $(+)$ and $10.3 \pm 1.24$ for Late $(+))$. Moreover in a direct choice between $\operatorname{Early}(+)$ vs. Late $(+)$ the rats consumed similar amounts of each (Fig. 2c, t[19] $=0.66$, $p=0.52)$. Rats also had no preference between $\operatorname{Early}(-)$ vs. Late $(-)$ flavors (Fig. $2 \mathrm{~d}, \mathrm{t}[19]=1.16, p=0.26$ ), showing that there was no inherent preference-enhancing advantage of experiencing a flavor early or late in a non-nutritive meal. This pattern of results supports the hypothesis that rats learn to prefer flavors occurring both early and late in a nutritive meal, contradicting the notion that the last flavor consumed in a meal should become more strongly associated with the meal's postingestive effects (at least as assessed when hungry).

\subsubsection{Two-bottle tests: Recently fed}

Lower levels of consumption overall in the recently fed tests compared to the Hungry tests was clearly evident, as would be expected, and provides a validity check to confirm the rats' behavior was sensitive to the recent feeding. When tested $90 \mathrm{~min}$ after the daily chow feeding, rats expressed a different pattern of preferences than they did when hungry (Fig. 3). Most notably, the Late $(+)$ flavor remained preferred relative to Late $(-$ ) (Fig. 3b, $\mathrm{t}[19]=3.67$, $p<0.002)$ but the $\operatorname{Early}(+)$ flavor was not preferred relative to Early $(-)$ (Fig. 3a, t $[19]=0.69, p=0.51)$. Although absolute intake of the Late $(+$ ) flavor was lower than it was when Hungry (compare Fig. 3b to Fig. 2b), the relative strength of the Late $(+)$ preference expressed as a percentage of overall intake (66.5\%) was similar to the Hungry test. But an apparent inconsistency is that the persistent preference for Late $(+)$ did not make it preferred in the choice vs. Early $(+)$, as intakes of these two were still similar (Fig. $3 c, t[19]=0.07, p=0.95$ ) in that test. Again there was no preference between the two $(-)$ flavors in a choice between them (Fig. $3 \mathrm{~d}, t[19]=1.31, p=0.21$ ).

Overall the fed two-bottle tests provide some support for the conclusion that learning about flavors encountered late in a nutritive meal may differ from learning that occurs early in the meal. This is based on the finding that the Late $(+)$ flavor remained preferred when tested in a replete state, while the $\operatorname{Early}(+)$ flavor did not. But this conclusion is tempered by the apparent lack of preference for Late $(+)$ over $\operatorname{Early}(+)$ when tested in this state.
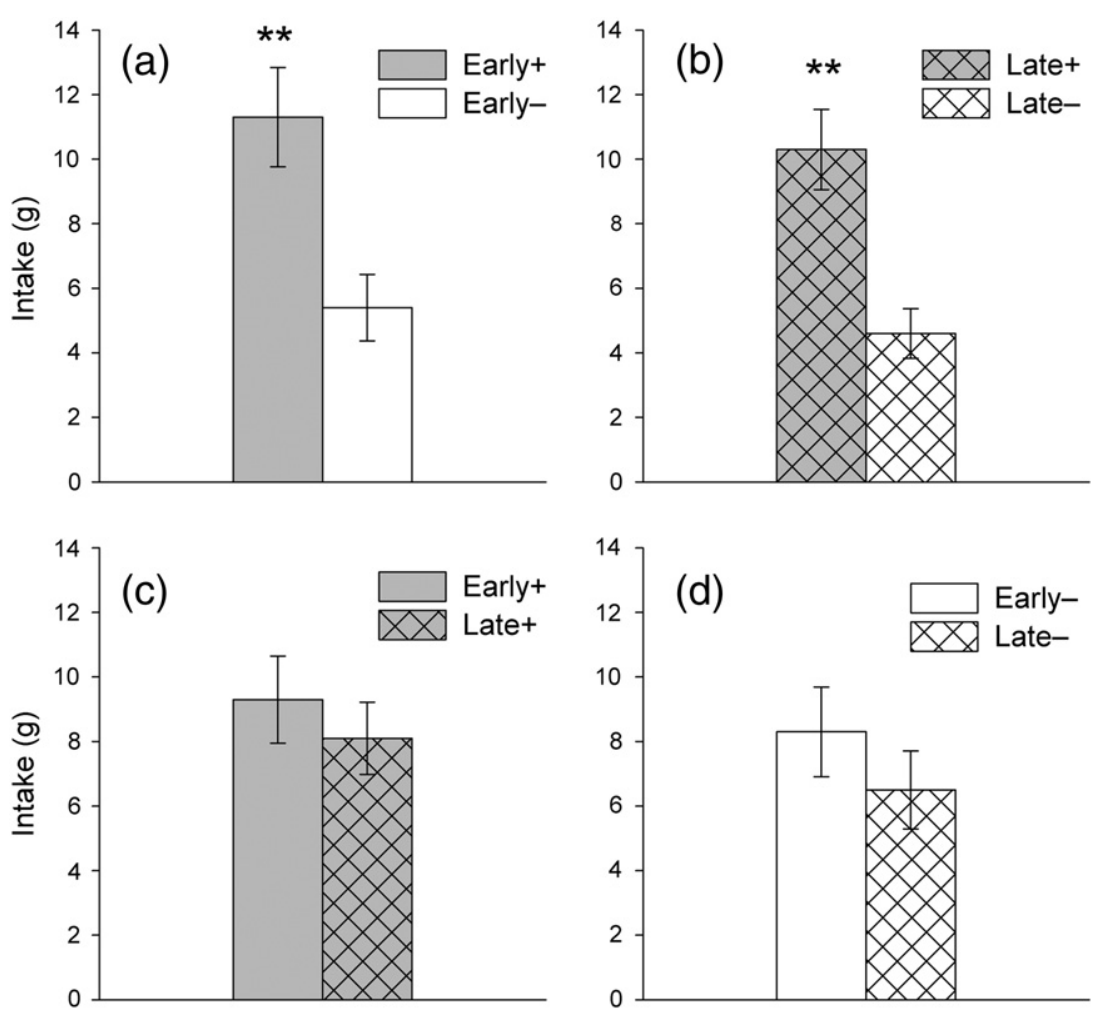

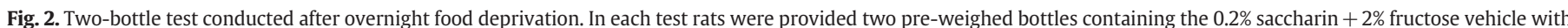

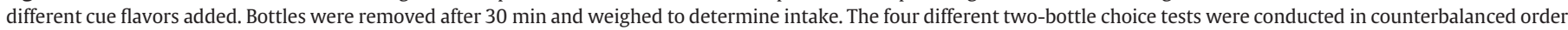

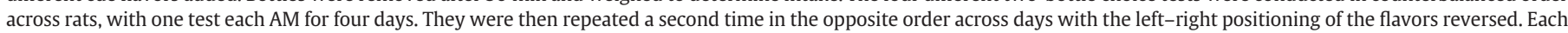
panel is the average of the two repetitions of that test. ${ }^{* *} p<0.01$; all other comparisons are not significant differences). 

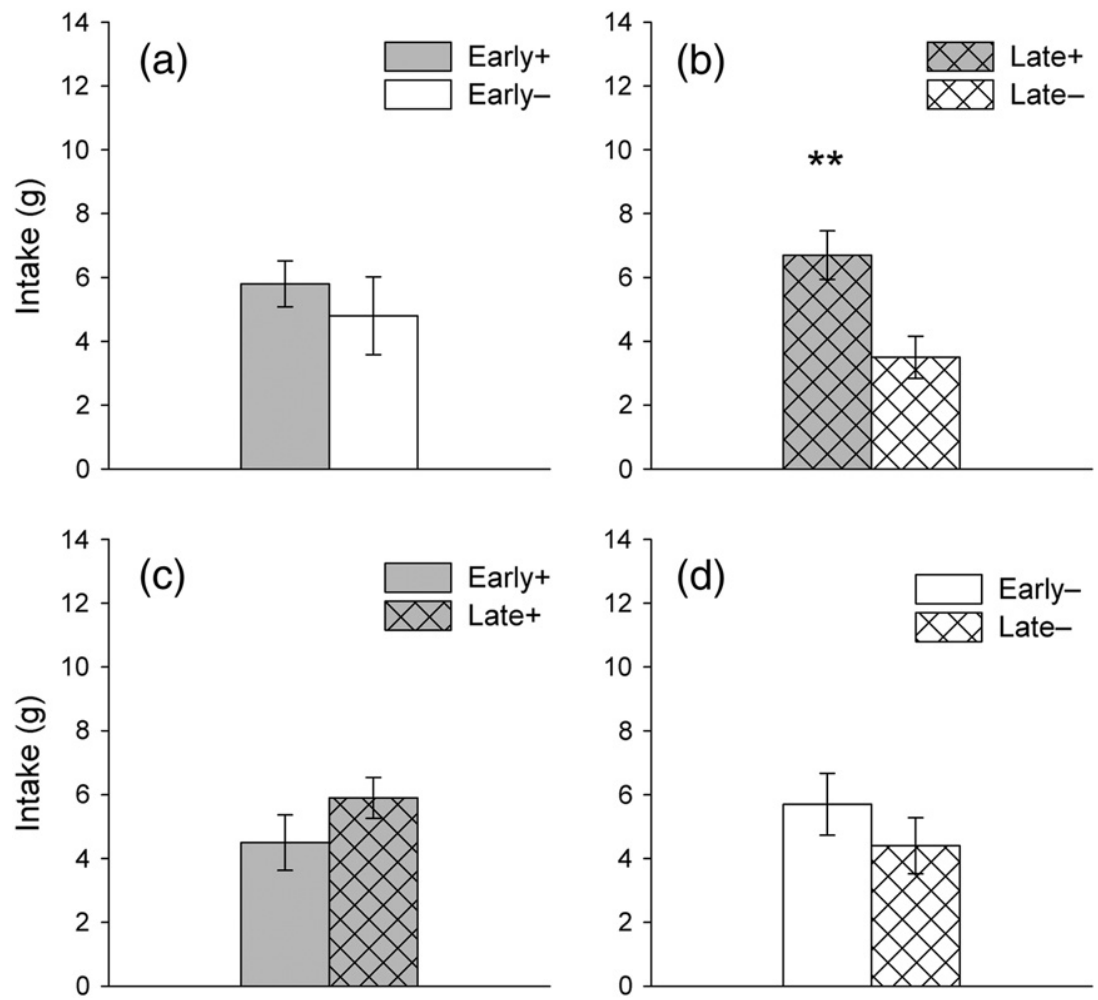

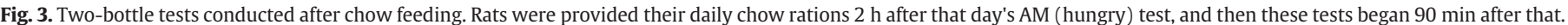

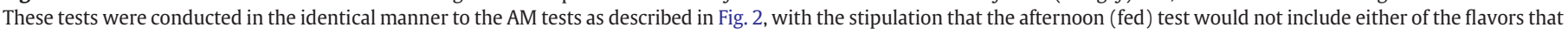
had appeared in that day's AM (hungry) test. $\left({ }^{* *} p<0.01\right.$; all other comparisons are not significant differences).

\section{Discussion}

There are two main findings of this study. First, rats learned to prefer flavors occurring in the first half and latter half of nutritive meals, relative to comparison flavors that were unpaired with nutrient. They did not appear to selectively associate only the early or late flavor with the postingestive nutrient consequences of the meal. Second, preferences for the $\operatorname{Early}(+)$ and Late $(+)$ flavor were differently sensitive to hunger state at the time of testing. When food deprived (similar to the state they would have been in at the start of a training session) rats had similarly strong preferences for both $\operatorname{Early}(+)$ and Late $(+)$ over their corresponding unpaired flavors. But when tested after a chow meal, they still expressed a strong preference for the Late $(+)$ flavor, but not the Early $(+)$ flavor. In other words, the expression of the learned Early flavor preference was hunger-state dependent while the learned Late flavor preference was not. Puzzlingly this was not reflected in a stronger preference for Late $(+)$ over Early $(+)$ in a direct choice between the two. Some possible reasons for that will be discussed below.

We believe that this experiment provides evidence that the physiological US for flavor-nutrient conditioning is detected within minutes of meal initiation, at least when glucose is the primary nutrient. If the postingestive US had a delayed onset, we would have expected to observe weak or no preference for $\operatorname{Early}(+)$, since Late $(+)$ would more closely coincide with the US. On the contrary, rats acquired a strong preference for both.

The alternative explanation that the early and late flavors are both remembered and become associated in the post-meal interval with a long-delayed postingestive US seems implausible for two reasons. First, it does not explain why the two preferences would be differently state-dependent. On the contrary, if the flavors both became associated with the US after the meal, we would expect any statedependence to be biased towards the replete state in which the associations were formed. Second, analogous experiments with learned aversions based on postingestive illness have typically not found equivalent learning about sequentially consumed flavors, instead finding that a flavor consumed more closely in time to onset of nausea actively interferes with learning about an earlier flavor [17]. We therefore interpret the rats' learned preference for the Early $(+)$ flavor in this experiment as evidence for rapid detection of a postingestive US before the late flavor is introduced.

Another observation that suggests rapid detection is that rats responded to the mid-session flavor switch differently in $(+)$ and $(-)$ training sessions, in a way that suggests "attribution" of postingestive effects to the Early $(+)$ flavor. They consumed much less of the Late $(+)$ flavor than we expected, and significantly less of it than the Late $(-)$ flavor. We based the session parameters on extensive pilot observations in an attempt to have rats consume similar amounts in the Early and Late halves, to equate familiarity. These efforts were not successful, but we believe that this pattern may reflect an underlying psychological process that has not been studied, as if rapid detection of nutritive consequences makes rats disinclined to switch flavors. It unlikely that smaller intake of Late $(+)$ was due to satiation. In the standard single-flavor training method rats typically begin consuming more in $(+)$ sessions than $(-)$ sessions, not less, even when higher nutrient concentrations are infused (for instance, $[5,6])$. Thus the pattern in the $(+)$ sessions suggests that rats become motivated to continue with a particular flavor when they sense nutrient onset. However this study was not designed to test that, so it remains to be explored.

Our conclusion that the US is detected early in the meal is consistent with experiments that have measured flavor-nutrient conditioning with glucose infused at different post-gastric sites [25]. In those experiments, only duodenal and jejunal infusions produced preference, implicating the proximal small intestine as a critical site for nutrient detection. It is thus physiologically plausible that a rewarding US signal could have fairly rapid onset. Gastric emptying begins rapidly after meal initiation and then slows with post-gastric negative feedback $[26,27]$. Based on parametric studies of this rapid 
initial emptying [27], we estimate that in our study in a typical $(+)$ session roughly $0.2-0.3 \mathrm{~g}$ of glucose (about $30 \%$ of the total glucose to be delivered in the session) would have emptied the stomach by the end of the Early segment. The entire length of the duodenum and jejunum would be filled within the first $10 \mathrm{~min}$ [28]. This is consistent with the idea of preabsorptive actions of glucose within the proximal intestines as the genesis of rewarding effects.

Yet absorption of glucose from a carbohydrate meal also commences quickly. Radio-labeled glucose ingested by fasted rats is detectable in circulation within 2 min of meal initiation [29]. Total glucose appearance rate in peripheral circulation accelerates to a peak within 6 min of an intragastric glucose load [30]. But the suggestion of a putative postabsorptive US is negated by the prior finding [25] that in a paradigm where duodenal or jejunal infusions are effective at producing preference for a paired flavor, ileal infusions of glucose are ineffective, as are hepatic portal infusions that mimic ordinary physiological uptake but are unaccompanied by intra-lumenal stimulation. Again this implicates a preabsorptive site of the earlyonset US within the proximal intestines. Yet post-absorptive sites may play a secondary role. While actions of glucose or other consequences of its metabolism at post-absorptive sites are generally found to be ineffective per se, (e.g., $[31,32]$ ) hepatic portal glucose can produce enhanced preference when accompanied by pre-absorptive stimulation [32,33]. Thus glucose sensing in the proximal small intestines may be necessary, but it may or may not be sufficient, and even then additional post-absorptive sites may be further involved in producing additional US effects.

Given the evidence that our rats learned a preference for the $\operatorname{Early}(+)$ flavor based on rapid detection of the nutrient, it requires some explanation as to why they would also learn a similarly strong preference for the Late $(+)$ if it actually followed US onset. A temporally backwards pairing would not be expected to create a strong association. This indicates the true nature of the postingestive US is likely more complex than a single signal arising from initial nutrient detection. It could be that the perceived US grows in intensity throughout the meal, and the Late $(+)$ flavor is associated with that increment. Indeed an effective postingestive US is detectable even when rats are already partially satiated and have nutrient already present in the GI tract prior to the flavor-nutrient pairing [34,35]. Rats can sense fairly small increments in nutrient delivered postingestively, since they learn to prefer flavors paired with dilute carbohydrate infusions (e.g. 1\% Polycose, $0.04 \mathrm{kcal} / \mathrm{ml}$ ) [36]. Thus they might prefer a late-occurring flavor based on its temporal association with increasing US intensity. But our results indicate something more complex since that process alone does not immediately explain the main difference in the $\operatorname{Early}(+)$ and Late $(+)$ preferences, which is that they were differently affected by deprivation state at testing.

Another possibility, then, is that the Early and Late flavors are becoming associated with qualitatively different USs. This could mean that in a meal of only one flavor rats actually learn multiple associations between the flavor and distinct postingestive USs, some acting early and some acting after a delay, with only some of those associations influenced by hunger. Recent work by Yiin et al. [34,35] regarding the influence of deprivation state on expression of flavornutrient conditioning has shown (using the more standard training paradigm with only a single flavor per training session) that rats trained to associate a single flavor with nutrition do not show a weaker preference for that flavor when tested under chow-replete conditions relative to chow-restricted tests. This is consistent with our findings if rats are indeed learning multiple associations about the nutrient-paired flavor. During the early part of a meal the flavor (experienced hungry) may become associated with rewarding effects of the initial, pre-absorptive detection of nutrients entering the proximal intestines. As the meal proceeds, or even after it ends, the flavor (now being experienced or remembered in a different state) becomes further associated with other, longer-delayed consequences of nutrient ingestion. This latter process, corresponding to our results with the Late $(+)$ flavor, would continue to promote intake regardless of deprivation state, potentially explaining why replete rats still prefer a nutrient-paired flavor, as has been shown [34,35].

The physiological basis of a late-acting US also remains to be identified. It may be a product of nutrient metabolism or one of many humoral satiety signals that would arise late in a meal and peak postprandially. For example, moderately low doses of CCK can condition learned preference for a paired non-nutritive flavor [37]. But devazepide does not attenuate the reinforcing effects of intraduodenal nutrient infusion [38], consistent with the suggestion that post-absorptive events like CCK's effects are secondary to nutrient detection in the proximal intestines. In any case, it is reasonable to suspect that the neural circuitry responding to postprandial satiety signals would be involved in flavor-nutrient conditioning. A principal way that external motivational factors influence intake is by modulating sensitivity to satiety [39]. One component of the adaptive response to flavor-nutrient learning, over and above increased "liking" of the CS flavor, is presumably an acquired "tolerance" of larger meals (after [40]). The early and late temporal portions of a meal roughly correspond to physiological events underlying satisfaction of hunger first, and then onset of a lasting satiety state thereafter. These may correspond to the qualitatively distinct unconditioned stimuli for learned preference that are suggested by this study.

There is an obvious caveat to our interpretation that rats learned qualitatively different, state-dependent associations about the Early $(+)$ and Late $(+)$ flavors. The $(+)$ vs. $(-)$ choice tests clearly indicated that the Late $(+)$ flavor remained preferred even after recent chow feeding while the $\operatorname{Early}(+)$ did not, but this was not reflected in the direct choice between $\operatorname{Early}(+)$ and Late $(+)$. Instead they were indifferent between the two $(+)$ flavors, which is difficult to explain if only the Late $(+)$ flavor is preferred while in that state. It is unlikely that either of the $(+)$ vs. ( - ) two-bottle test results is an artifact of differential evaluation of the Early $(-)$ and Late $(-)$ flavors, as rats were always indifferent between those two. One possibility is based on evidence that $(-)$ flavors themselves acquire inhibitory control over intake due to their explicitly unpaired relationship with the nutrient US $[41,42]$. Conceivably the Late $(+)$ flavor, because it is still preferred in the non-deprived state, might also stimulate a number of generalized appetitive responses that carry over to enhance intake of the Early $(+)$ even though that flavor would not by itself be preferred in that state. These putative generalized appetitive effects are plausible given the evidence that conditioned cues associated with preferred food can also stimulate intake of less preferred foods [43]. But the same carryover effect would not promote intake of either (-) flavor if they had acquired inhibitory effects, as has been suggested. This could cause a strong Late $(+)$ preference to be seen in a Late $(+)$ vs. Late $(-)$ test but obscured in a Late $(+)$ vs. $\operatorname{Early}(+)$ test. Admittedly this is rather speculative, but we offer it as one plausible explanation of why the unusual Late $(+)$ vs. $\operatorname{Early}(+)$ result does not invalidate the conclusion that the Late $(+)$ flavor alone remains preferred in the non-deprived state. Nonetheless, replication and additional study will be useful for clarifying this observation.

This experiment also directly tests the proposal (after $[1,18]$ ) that stronger preferences would be learned for flavors at the end of the meal, which has been offered as an explanation for how foods routinely eaten as desserts become especially well liked. But we find no indication that rats more strongly associate the Late $(+)$ flavor with nutrition. Moreover, the preference for the $\operatorname{Early}(+)$ flavor is in itself substantial evidence against the idea that postingestive US provided by foods eaten early in the meal is experienced after a significant delay. However, the possible influence of such sequential effects in producing weaker or stronger preferences for flavors eaten at different points in the meal should not be dismissed based on these results. First, the postingestive US in flavor-nutrient may vary 
depending on the macronutrient composition of the meal. This experiment used glucose, but other carbohydrates, fats, proteins and even ethanol can be effective in flavor-nutrient conditioning. But, for instance, learned preference for a flavor paired with fat requires more training and is ultimately weaker than the preference for a carbohydrate-paired flavor $[13,44]$, and this may indicate that fat involves a different US with slower onset. Therefore meals of some macronutrient composition, particularly those high in fat or complex carbohydrates, might indeed bias flavor-nutrient conditioning to promote learning about flavors late in the meal. Additional experiments comparing learning about early vs. late flavors when different nutrients are used may provide additional insight into common or differing mechanisms of action for postingestive reinforcing effects of different macronutrients.

Also, while this experiment did not support the idea that there would be stronger preferences for late flavors, it did find an effect of deprivation state that might offer insight into why 'entrée' flavors may be especially attractive only when hungry, while 'dessert' flavors remain attractive in the absence of hunger. In humans' eating habits at least, it seems the very essence of a dessert is that it can renew interest in eating and remains especially palatable even after a satiating meal. Another potential influence that could contribute to the dessert effect' previously proposed $[1,18]$ is that desserts (at least in Western cuisines) are typically much sweeter than anything else in the meal. This adds the role of flavor-flavor associations in enhancing liking of other flavors in the dessert, but there is also evidence that sweetening can promote flavor-nutrient conditioning [11], perhaps psychologically through an attentional/memorial effect or physiologically through enhanced cephalic phase responses that promote rapid and efficient handling of nutrients postingestively. These influences, rather than its position at the end of a meal, could explain how flavor-nutrient conditioning promotes especially strong liking of dessert flavors.

In conclusion, this evidence of reinforcing events acting on more than one time course adds to the growing evidence for multiple distinct or interacting sites of postingestive nutrient action that support flavor-nutrient conditioning. Several authors have proposed that learned positive responses to flavors can recruit multiple, independent psychological processes, and this learning can lead to increased intake through a number of behavioral changes. These have variously been described in the context of several theoretical frameworks as appetitive vs. consummatory $[45,46]$, hedonic vs. expectancy $[23,47,48]$, liked vs. beneficial [49], sensory vs. evaluative [50,51], and liked vs. wanted [[6], after [52]]. Each of these approaches has considerable merit, since the true nature of how associative learning alters flavor evaluation and intake is likely quite complex. We now suggest that isolating and dissociating the learning mechanisms operating early or late in the meal and post-meal interval may be a useful strategy for illuminating the organization of learned preference behavior.

\section{Acknowledgments}

A thesis discussing preliminary results of this project was submitted by MCW to Bucknell University as part of the requirements for graduation with Honors in Animal Behavior. We appreciate the feedback provided during that phase of the work by Drs. Peter Judge and Tristan Stayton. We thank Dr. Andrea Halpern for helpful feedback on an earlier draft of this manuscript.

\section{References}

[1] Capaldi ED. Conditioned food preferences. In: Capaldi ED, editor. Why we eat what we eat: the psychology of eating. Washington, DC, US: American Psychological Association; 1996. p. 53-80.
[2] Sclafani A. Nutritionally based learned flavor preferences in rats. In: Capaldi ED, Powley TL, editors. Taste, experience, and feeding. Washington, DC, US: American Psychological Association; 1990. p. p139-56.

[3] Sclafani A. Macronutrient-conditioned flavor preferences. In: Berthoud $\mathrm{H}$, Seeley R, editors. Neural and metabolic control of macronutrient intake. Boca Raton, FL: CRC Press; 1999. p. p93-p107.

[4] Zellner DA. How foods get to be liked: some general mechanisms and some special cases. In: Bolles RC, editor. The hedonics of taste. Hillsdale, NJ: Lawrence Erlbaum Associates, Inc.; 1991. p. 199-217.

[5] Perez C, Lucas F, Sclafani A. Increased flavor acceptance and preference conditioned by the postingestive actions of glucose. Physiol Behav 1998;64: 483-92.

[6] Myers KP, Sclafani A. Conditioned enhancement of flavor evaluation reinforced by intragastric glucose: I. intake acceptance and preference analysis. Physiol Behav 2001;74:481-93.

[7] Sclafani A. How food preferences are learned: laboratory animal models. Proc Nutr Soc 1995;54:419-27

[8] Warwick ZS, Weingarten HP. Dissociation of palatability and calorie effects in learned flavor preferences. Physiol Behav 1994;55:501-4.

[9] Yeomans MR, Leitch M, Gould NJ, Mobini S. Differential hedonic, sensory and behavioral changes associated with flavor-nutrient and flavor-flavor learning. Physiol Behav 2008;93:798-806.

[10] Drucker DB, Ackroff K, Sclafani A. Flavor preference produced by intragastric polycose infusions in rats using a concurrent conditioning procedure. Physiol Behav 1993;54:351-5

[11] Ackroff K. Learned flavor preferences. The variable potency of post-oral nutrient reinforcers. Appetite 2008;51:743-6.

[12] Sclafani A, Cardieri C, Tucker K, Blusk D, Ackroff K. Intragastric glucose but not fructose conditions robust flavor preferences in rats. Am J Physiol 1993;265:R320-5.

[13] Ackroff K, Dym C, Yiin YM, Sclafani A. Rapid acquisition of conditioned flavor preferences in rats. Physiol Behav 2009;97:406-13.

[14] Boakes RA, Lubart T. Enhanced preference for a flavour following reversed flavour-glucose pairing. Q J Exp Psychol B 1988;40:49-62.

[15] Elizalde G, Sclafani A. Starch-based conditioned flavor preferences in rats: influence of taste, calories and CS-US delay. Appetite 1988;11:179-200.

[16] Bernstein IL, Vitiello MV, Sigmundi RA. Effects of interference stimuli on the acquisition of learned aversions to foods in the rat. J Comp Physiol Psychol 1980;94:921-31.

[17] Revusky S. The role of interference in association over a delay. In: Honig WK, James PHR, editors. Animal memory. New York: Academic Press; 1971.

[18] Capaldi ED, Campbell DH, Sheffer JD, Bradford JP. Non-reinforcing effects of giving "dessert" in rats. Appetite 1987;9:99-112.

[19] Rozin P, Levine E, Stoess C. Chocolate craving and liking. Appetite 1991;17: 199-212.

[20] Davis JD, Campbell CS. Chronic intrajugular, intraportal, gastric, and duodenal cannulae for the rat. In: Singh D, Avery DD, editors. Physiological techniques in behavioral research. Monterey, CA: Brooks Cole; 1975. p. 163-77.

[21] Ackroff K, Touzani K, Peets TK, Sclafani A. Flavor preferences conditioned by intragastric fructose and glucose: differences in reinforcement potency. Physiol Behav 2001:72:691-703.

[22] Sclafani A, Fanizza LJ, Azzara AV. Conditioned flavor avoidance, preference, and indifference produced by intragastric infusions of galactose, glucose, and fructose in rats. Physiol Behav 1999;67:227-34

[23] Drucker DB, Ackroff K, Sclafani A. Nutrient-conditioned flavor preference and acceptance in rats: effects of deprivation state and nonreinforcement. Physiol Behav 1994;56:701-7.

[24] Elizalde G, Sclafani A. Flavor preferences conditioned by intragastric polycose infusions: a detailed analysis using an electronic esophagus preparation. Physiol Behav 1990;47:63-77.

[25] Ackroff K, Yiin YM, Sclafani A. Post-oral infusion sites that support glucoseconditioned flavor preferences in rats. Physiol Behav 2010;99:402-11.

[26] Brener W, Hendrix TR, McHugh PR. Regulation of the gastric emptying of glucose. Gastroenterology 1983;85:76-82.

[27] Kaplan JM, Spector AC, Grill HJ. Dynamics of gastric emptying during and after stomach fill. Am J Physiol 1992;263:R813-9.

[28] Wiepkema PR, Prins AJ, Steffens AB. Gastrointestinal food transport in relation to meal occurrence in rats. Physiol Behav 1972;9:759-63.

[29] Steffens A. Rapid absorption of glucose in the intestinal tract of the rat after ingestion of a meal. Physiol Behav 1969;4:829-32.

[30] Smadja C, Morin J, Ferre P, Girard J. Metabolic fate of a gastric glucose load in unrestrained rats bearing a portal vein catheter. Am J Physiol 1988;254:E407-13.

[31] Gowans SE, Weingarten HP. Elevations of plasma glucose do not support taste-topostingestive consequence learning. Am J Physiol 1991;261:R1409-17.

[32] Tordoff MG, Friedman MI. Hepatic portal glucose infusions decrease food intake and increase food preference. Am J Physiol 1986;251:R192-6.

[33] Revusky SH, Smith Jr MH, Chalmers DV. Flavor preference: effects of ingestioncontingent intravenous saline or glucose. Physiol Behav 1971;6:341-3.

[34] Yiin YM, Ackroff K, Sclafani A. Flavor preferences conditioned by intragastric nutrient infusions in food restricted and free-feeding rats. Physiol Behav 2005;84: 217-31.

[35] Yiin YM, Ackroff K, Sclafani A. Food deprivation enhances the expression but not acquisition of flavor acceptance conditioning in rats. Appetite 2005;45:152-60.

[36] Ackroff K, Sclafani A. Flavor preferences conditioned by intragastric infusions of dilute polycose solutions. Physiol Behav 1994;55:957-62.

[37] Perez C, Sclafani A. Cholecystokinin conditions flavor preferences in rats. Am J Physiol 1991;260:R179-85. 
[38] Perez C, Lucas F, Sclafani A. Devazepide, a CCK(A) antagonist, attenuates the satiating but not the preference conditioning effects of intestinal carbohydrate infusions in rats. Pharmacol Biochem Behav 1998;59:451-7.

[39] Woods SC. Gastrointestinal satiety signals I. An overview of gastrointestinal signals that influence food intake. Am J Physiol Gastrointest Liver Physiol 2004;286:G7-13.

[40] Woods SC. The eating paradox: how we tolerate food. Psychol Rev 1991;98:488-505.

[41] Harris JA, Shand FL, Carroll LQ, Westbrook RF. Persistence of preference for a flavor presented in simultaneous compound with sucrose. J Exp Psychol Anim Behav Process 2004;30:177-89.

[42] Boakes RA, Colagiuri B, Mahon M. Learned avoidance of flavors signaling reduction in a nutrient. J Exp Psychol Anim Behav Process 2010;36:117-25.

[43] Boggiano MM, Dorsey JR, Thomas JM, Murdaugh DL. The Pavlovian power of palatable food: lessons for weight-loss adherence from a new rodent model of cue-induced overeating. Int J Obes (Lond) 2009;33:693-701.

[44] Revelle $\mathrm{CH}$, Warwick ZS. Flavor-nutrient learning is less rapid with fat than with carbohydrate in rats. Physiol Behav 2009;97:381-4.

[45] Myers KP, Hall WG. Evidence that oral and nutrient reinforcers differentially condition appetitive and consummatory responses to flavors. Physiol Behav 1998;64:493-500.
[46] Myers KP, Hall WG. Conditioned changes in appetitive and consummatory responses to flavors paired with oral or nutrient reinforcement among adult rats. Physiol Behav 2000;68:603-10.

[47] Capaldi ED, Sheffer JD. Contrast and reinforcement in consumption. Learn Motiv 1992;23:63-79.

[48] Fedorchak PM, Bolles RC. Hunger enhances the expression of calorie- but not taste-mediated conditioned flavor preferences. J Exp Psychol Anim Behav Process 1987;13:73-9.

[49] Rozin P, Zellner D. The role of Pavlovian conditioning in the acquisition of food likes and dislikes. Ann NY Acad Sci 1985;443:189-202.

[50] Yeomans MR, Mobini S, Elliman TD, Walker HC, Stevenson RJ. Hedonic and sensory characteristics of odors conditioned by pairing with tastants in humans. J Exp Psychol Anim Behav Process 2006;32:215-28.

[51] Yeomans MR, Mobini S. Hunger alters the expression of acquired hedonic but not sensory qualities of food-paired odors in humans. J Exp Psychol Anim Behav Process 2006;32:460-6.

[52] Berridge KC. Food reward: brain substrates of wanting and liking. Neurosci Biobehav Rev 1996;20:1-25. 